\title{
Chiral sum rules and vacuum condensates from tau-lepton decay data
}

\author{
C.A. Dominguez, ${ }^{a, b}$ L.A. Hernandez, ${ }^{a, b}$ K. Schilcher ${ }^{a, b, c}$ and H. Spiesberger ${ }^{a, b, c}$ \\ ${ }^{a}$ Centre for Theoretical and Mathematical Physics, and Department of Physics, \\ University of Cape Town, Rondebosch 7700, South Africa \\ ${ }^{b}$ National Institute of Theoretical Physics, \\ Private Bag X1, Matieland 7602, South Africa \\ ${ }^{c}$ PRISMA Cluster of Excellence, Institut für Physik, \\ Johannes Gutenberg-Universität, D-55099 Mainz, Germany \\ E-mail: cesareo.dominguez@uct.ac.za, HRNLUI001@myuct.ac.za, \\ karl.schilcher@uni-mainz.de, spiesber@uni-mainz.de
}

ABSTRACT: QCD finite energy sum rules, together with the latest updated ALEPH data on hadronic decays of the tau-lepton are used in order to determine the vacuum condensates of dimension $d=2$ and $d=4$. These data are also used to check the validity of the Weinberg sum rules, and to determine the chiral condensates of dimension $d=6$ and $d=8$, as well as the chiral correlator at zero momentum, proportional to the counter term of the $\mathcal{O}\left(p^{4}\right)$ Lagrangian of chiral perturbation theory, $\bar{L}_{10}$. Suitable (pinched) integration kernels are introduced in the sum rules in order to suppress potential quark-hadron duality violations. We find no compelling indications of duality violations in the kinematic region above $s \simeq 2.2 \mathrm{GeV}^{2}$ after using pinched integration kernels.

KeYwords: Sum Rules, QCD

ARXIV EPRINT: 1410.3779 


\section{Contents}

1 Introduction 1

$2 \quad$ QCD finite energy sum rules and vacuum condensates 2

$\begin{array}{llr}3 & \text { Chiral sum rules and chiral vacuum condensates } & 8\end{array}$

4 Conclusion

\section{Introduction}

Experimental data on hadronic decays of the $\tau$-lepton [1-4] play an essential role in the determination of several QCD quantities [5]. For instance, the $R_{\tau}$-ratio provides the cleanest determination of the running strong coupling at the scale of the $\tau$-mass. In addition, these data have been used in the past to extract the values of some of the QCD vacuum condensates entering the operator product expansion (OPE) of current correlators at short distances beyond perturbation theory [6-17]. This OPE is one of the two fundamental pillars of the method of QCD sum rules, the other being the assumption of quark-hadron duality [18]. The latter allows to relate QCD with hadronic physics by means of Cauchy's theorem in the complex squared energy plane. A key advantage of hadronic $\tau$-decay data is that it determines both the vector and the axial-vector spectral functions. This feature allows to check the saturation of a variety of chiral sum rules [13, 19-21], as well as to determine the chiral correlator at zero momentum [5, 21-27], proportional to the counter term of the order $\mathcal{O}\left(p^{4}\right)$ Lagrangian of chiral perturbation theory (CHPT), $\bar{L}_{10}$. It also allows for a determination of the chiral condensates of dimension $d=6$ and $d=8[5,13,21-27]$.

Most of these past determinations made use of the hadronic spectral functions in the vector and axial-vector channel as measured by the ALEPH Collaboration [2,3]. This data base was known to be problematic due to the incompleteness of the data correlations [28], thus casting some doubt on the uncertainties in results obtained using these data. A new ALEPH data set has recently become available [4], with the data organized in different bins, and with a corrected error correlation matrix. In this paper we employ these data to revisit the vacuum condensate determinations, the saturation of chiral sum rules, and the determination of $\bar{L}_{10}$ and the chiral condensates of dimension $d=6$ and $d=8$. The procedure is based on finite energy QCD sum rules (FESR), weighted with suitable integration kernels to account for potential duality violations (DV). Our results mostly confirm central values obtained previously using the original ALEPH data base, with uncertainties being slightly higher in some cases, and lower in others. 


\section{QCD finite energy sum rules and vacuum condensates}

We consider the (charged) vector and axial-vector current correlators

$$
\begin{aligned}
\Pi_{\mu \nu}^{V V}\left(q^{2}\right) & =i \int d^{4} x e^{i q x}\left\langle 0\left|T\left(V_{\mu}(x) V_{\nu}^{\dagger}(0)\right)\right| 0\right\rangle \\
& =\left(-g_{\mu \nu} q^{2}+q_{\mu} q_{\nu}\right) \Pi_{V}\left(q^{2}\right), \\
\Pi_{\mu \nu}^{A A}\left(q^{2}\right) & =i \int d^{4} x e^{i q x}\left\langle 0\left|T\left(A_{\mu}(x) A_{\nu}^{\dagger}(0)\right)\right| 0\right\rangle \\
& =\left(-g_{\mu \nu} q^{2}+q_{\mu} q_{\nu}\right) \Pi_{A}\left(q^{2}\right)-q_{\mu} q_{\nu} \Pi_{0}\left(q^{2}\right),
\end{aligned}
$$

where $V_{\mu}(x)=: \bar{u}(x) \gamma_{\mu} d(x):, A_{\mu}(x)=: \bar{u}(x) \gamma_{\mu} \gamma_{5} d(x):$, with $u(x)$ and $d(x)$ the quark fields, and $\Pi_{V, A}\left(q^{2}\right)$ normalized in perturbative QCD (PQCD) (in the chiral limit) according to

$$
\frac{1}{\pi} \operatorname{Im} \Pi_{V}^{P Q C D}(s)=\frac{1}{\pi} \operatorname{Im} \Pi_{A}^{P Q C D}(s)=\frac{1}{4 \pi^{2}}\left(1+\frac{\alpha_{s}(s)}{\pi}+\ldots\right),
$$

where $s \equiv q^{2}>0$ is the squared energy. Lorentz decomposition is used to separate the correlation function into its $J=1$ and $J=0$ parts. To the accuracy needed in the following, the vector current can be assumed to be conserved. The correlators are well-known up to five-loop order [29-33]. Solving the renormalization group equation for the strong coupling, one can express $\alpha_{s}(s)$ in terms of the coupling at a given scale $s_{0}$, with the result at six-loop order being [12]

$$
\begin{aligned}
a_{s}(s)= & a_{s}\left(s_{0}\right)+a_{s}^{2}\left(s_{0}\right)\left(\frac{1}{2} \beta_{1} \eta\right)+a_{s}^{3}\left(s_{0}\right)\left(\frac{1}{2} \beta_{2} \eta+\frac{1}{4} \beta_{1}^{2} \eta^{2}\right) \\
& +a_{s}^{4}\left(s_{0}\right)\left[\frac{1}{2} \beta_{3} \eta+\frac{5}{8} \beta_{1} \beta_{2} \eta^{2}+\frac{1}{8} \beta_{1}^{3} \eta^{3}\right] \\
& +a_{s}^{5}\left(s_{0}\right)\left[-b_{3} \eta+\frac{3}{8} \beta_{2}^{2} \eta^{2}+\frac{3}{4} \beta_{1} \beta_{3} \eta^{2}+\frac{13}{24} \beta_{1}^{2} \beta_{2} \eta^{3}+\frac{1}{16} \beta_{1}^{4} \eta^{4}\right]
\end{aligned}
$$

with

$$
\eta=\ln \left(\frac{s}{s_{0}}\right) .
$$

The coefficients of the $\beta$-function are given by

$$
\begin{aligned}
& \beta_{1}=-\frac{1}{2}\left(11-\frac{2}{3} n_{F}\right), \quad \beta_{2}=-\frac{1}{8}\left(102-\frac{38}{3} n_{F}\right), \\
& \beta_{3}=-\frac{1}{32}\left(\frac{2857}{2}-\frac{5033}{18} n_{F}+n_{F}^{2}\right),
\end{aligned}
$$

and

$b_{3}=\frac{1}{4^{4}}\left[\frac{149753}{6}+3564 \zeta_{3}-\left(\frac{1078361}{162}+\frac{6508}{27} \zeta_{3}\right) n_{F}+\left(\frac{50065}{162}+\frac{6472}{81} \zeta_{3}\right) n_{F}^{2}+\frac{1093}{729} n_{F}^{3}\right]$,

with $\zeta_{3}=1.202$. 
Non-perturbative contributions are parametrized in terms of the vacuum condensates entering the OPE

$$
\left.4 \pi^{2} \Pi\left(Q^{2}\right)\right|_{V, A}=\left.\sum_{N=1}^{\infty} \frac{1}{Q^{2 N}} C_{2 N}\left(Q^{2}, \mu^{2}\right)\left\langle O_{2 N}\left(\mu^{2}\right)\right\rangle\right|_{V, A},
$$

where $Q^{2}=-q^{2}$, and $\mu$ is a renormalization scale separating long distance non-perturbative effects associated with the vacuum condensates $\left\langle O_{2 N}\left(\mu^{2}\right)\right\rangle$ from the short distance effects which are encapsulated in the Wilson coefficients $C_{2 N}\left(Q^{2}, \mu^{2}\right)$. In principle, the lowest dimension is $d=4$ as there are no gauge invariant operators of dimension $d=2$ in QCD. However, the absence of such a condensate will be confirmed by the results of this analysis. At dimension $d=4$, and in the chiral limit, the only contribution is from the (chiralsymmetric) gluon condensate

$$
\left.C_{4}\left\langle O_{4}\right\rangle\right|_{V, A}=\frac{\pi^{2}}{3}\left\langle\frac{\alpha_{s}}{\pi} G_{\mu \nu} G^{\mu \nu}\right\rangle
$$

where $\alpha_{s}$ is the running strong coupling, and in the sequel $\left\langle 0\left|O_{2 N}\right| 0\right\rangle \equiv\left\langle O_{2 N}\right\rangle$ is to be understood. This condensate is renormalization group invariant to all orders in PQCD (in the chiral limit).

Invoking Cauchy's theorem in the complex squared energy s-plane, and assuming (global) quark-hadron duality leads to the FESR

$$
-\left.\frac{1}{2 \pi i} \oint_{|s|=s_{0}} d s f(s) \Pi(s)\right|_{V, A} ^{\mathrm{QCD}}=\int_{0}^{s_{0}} d s f(s) \rho_{V, A}(s),
$$

where $f(s)$ is an integration kernel and $\rho_{V, A}(s)$ are the hadronic spectral functions,

$$
\rho_{V, A}(s)=\left.\frac{1}{\pi} \operatorname{Im} \Pi(s)\right|_{V, A} ^{\mathrm{HAD}}=\frac{1}{2 \pi^{2}}[v(s), a(s)]_{\mathrm{ALEPH}}
$$

provided by the ALEPH data. Since PQCD is not applicable on the positive real $s$-axis, a very early warning against the unqualified use of sum rules was raised [34] even before the QCD sum rule program was proposed. A priori it is not clear at which scale duality sets in. It was shown $[19,35]$ that by reducing the impact of $\left.\Pi(s)\right|_{V, A} ^{\mathrm{QCD}}$ in the contribution of the integration contour near the positive real axis in eq. (2.10) by a suitable integration kernel $f(s)$ (pinching), the range of manifest duality can be increased substantially. In particular, we have shown previously for the old ALEPH data that there is clear evidence that duality is satisfied towards the end of the decay spectrum [17]. In practice, the absence of (DV) can be inferred from sum rules where their values are known from other sources or, less compelling, from the stability of the integral against variations of the upper limit of integration $s_{0}$. We will demonstrate below that duality can be observed with the new ALEPH data for many sum rules. However, DV is a contentious issue relying on specific models $[22,23,25]$, as discussed in more detail in section 3.

The contour integral in eq. (2.10) is usually computed using fixed order perturbation theory (FOPT) or contour improved perturbation theory (CIPT). In the former case the strong coupling is frozen at a scale $s_{0}$ and the renormalization group (RG) is implemented 
after integration. In CIPT $\alpha_{s}(s)$ is running and the RG is used before integrating, thus requiring solving numerically the $\mathrm{RG}$ equation for $\alpha_{s}(s)$ at each point on the integration contour. In the specific case of the determination of the vacuum condensates we found CIPT to be superior to FOPT in that results turn out to be more stable as a function of $s_{0}$. To implement CIPT it is convenient to introduce the Adler function

$$
D(s) \equiv-s \frac{d}{d s} \Pi(s),
$$

with $\Pi(s) \equiv \Pi_{V, A}(s)$. Invoking Cauchy's theorem and after integration by parts the following relation is obtained

$$
\oint_{|s|=s_{0}} d s\left(\frac{s}{s_{0}}\right)^{N} \Pi(s)=\frac{1}{N+1} \frac{1}{s_{0}^{N}} \oint_{|s|=s_{0}} \frac{d s}{s}\left(s^{N+1}-s_{0}^{N+1}\right) D(s) .
$$

After RG improvement, the perturbative expansion of the Adler function becomes

$$
D(s)=\frac{1}{4 \pi^{2}} \sum_{m=0} K_{m}\left[\frac{\alpha_{s}(-s)}{\pi}\right]^{m},
$$

where [29-33] $K_{0}=K_{1}=1, K_{2}=1.6398, K_{3}=6.3710$, for three flavours, and $K_{4}=$ 49.076 [36]. The vacuum condensates are then determined from the pinched FESR

$$
\begin{aligned}
C_{2 N+2}\left\langle O_{2 N+2}\right\rangle= & (-)^{N+1} 4 \pi^{2} s_{0}^{N} \int_{0}^{s_{0}} d s\left[1-\left(\frac{s}{s_{0}}\right)^{N}\right] \frac{1}{\pi} \operatorname{Im} \Pi(s)^{\mathrm{HAD}} \\
& +(-)^{N} s_{0}^{N+1}\left[M_{0}\left(s_{0}\right)-M_{N}\left(s_{0}\right)\right],
\end{aligned}
$$

where the moments $M_{N}\left(s_{0}\right)$ are given by

$$
M_{N}\left(s_{0}\right)=\frac{1}{2 \pi} \frac{1}{(N+1)} \sum_{m=0} K_{m}\left[I_{N+1, m}\left(s_{0}\right)-I_{0, m}\left(s_{0}\right)\right],
$$

with

$$
I_{N, m} \equiv i \oint_{|s|=s_{0}} d s\left(\frac{s}{s_{0}}\right)^{N}\left[\frac{\alpha_{s}(-s)}{\pi}\right]^{m}
$$

The latest ALEPH data compilation [4] includes the vector and axial-vector channels separately, as well as their sum. Their data are given in tables for the normalised invariant mass-squared distributions. We determine the spectral functions as described, for example, in [3] and approximate the sum rule integrals by sums over bins, taking into account the corrected correlation matrix. We should note that we will omit the last two points with the highest $s$ values in the figures for our results to be discussed below; they have very large experimental uncertainties and do not affect our conclusions. We use the following values for the input parameters

$$
\begin{aligned}
m_{\pi} & =139.57018(35) \mathrm{MeV}, \\
f_{\pi} & =92.21(14) \mathrm{MeV}, \\
M_{\tau} & =1776.82(16) \mathrm{MeV}, \\
V_{u d} & =0.97425(22), \\
S_{\mathrm{EW}} & =1.0198, \quad B_{e}=0.17818 .
\end{aligned}
$$




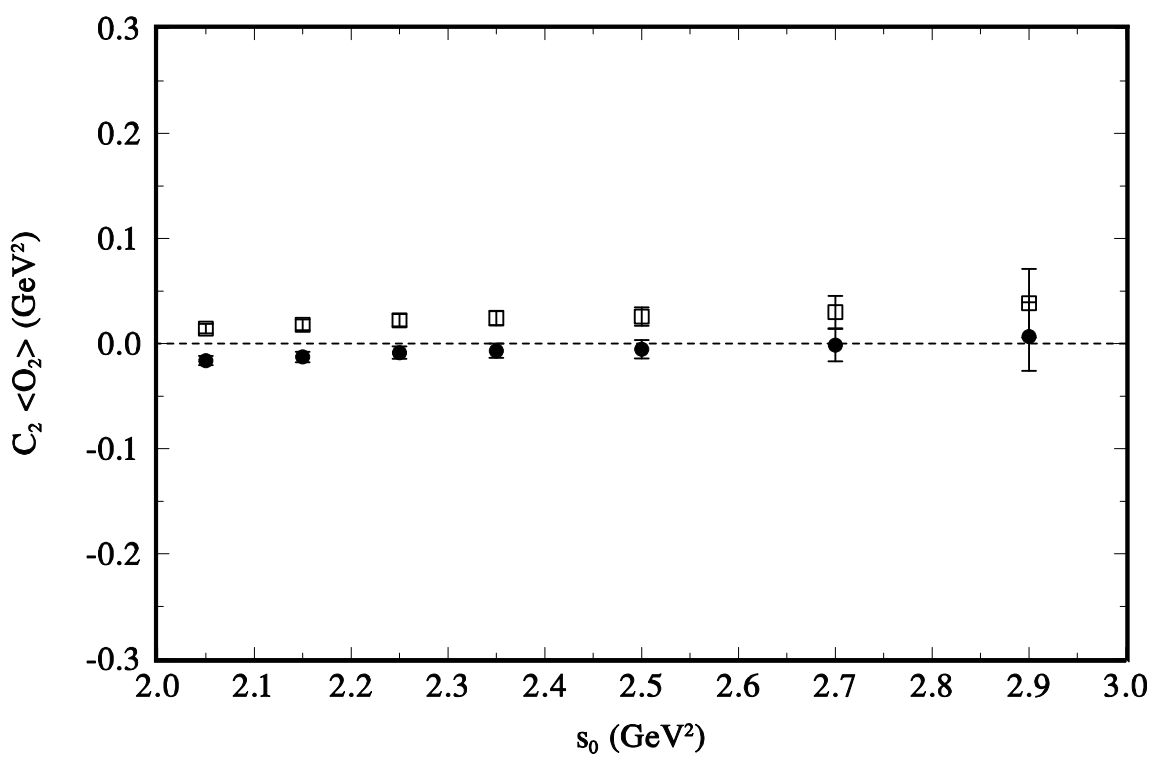

Figure 1. The dimension $d=2$ condensate in CIPT from the FESR, eq. (2.15), with $N=0$. The ALEPH data for the $V+A$ spectral function was used, and the resulting condensate divided by 2 . The two sets of points correspond to $\alpha_{s}=0.354$ (full dots) and $\alpha_{s}=0.328$ (open squares).

The first four values are taken from the particle data group [37]. $S_{\mathrm{EW}}$ is needed to include the renormalization-group improved electroweak corrections [38]. As the leptonic branching ratio $B_{e}$ was not updated in the recent paper [3], we again use the one given in the earlier ALEPH report [2]. From the latest analysis [5], we have $\alpha_{s}\left(M_{\tau}^{2}\right)=0.341 \pm 0.013$ in CIPT and $\alpha_{s}\left(M_{\tau}^{2}\right)=0.319 \pm 0.014$ in FOPT. For consistency we use the CIPT result in the following.

Proceeding with the determination of a potential $d=2$ condensate (presumably chiralsymmetric) we have used the data base for $V+A$ in the FESR and divided the answer by a factor two. In figure 1 we show the result in the stability region. The solid dots correspond to the minimum value of $\alpha_{s}$ and the open squares to its maximum value. As expected, this $d=2$ term is consistent with zero. Notice that in this case there is no pinching integration kernel as $N=0$ in eq. (2.15).

Next, we make use of this result and consider the pinched FESR, eq. (2.15), with $N=1$. The condensate of $d=4$ is shown in figure 2 , for $V, A$ and $\frac{1}{2}(V+A)$. We observe that for $s_{0} \gtrsim 2.2 \mathrm{GeV}^{2}$ and within errors

$$
C_{4}\left\langle O_{4}\right\rangle_{V}=C_{4}\left\langle O_{4}\right\rangle_{A}=C_{4}\left\langle O_{4}\right\rangle_{\frac{1}{2}(V+A)}
$$

over a wide range of $s_{0}$. This equality is an essential result of QCD. In addition the $d=4$ condensate is generally expected to be positive because it is dominated by the gluon condensate, eq. (2.9), which in turn is directly related to the vacuum energy density [39, 40],

$$
\varepsilon=\frac{\pi}{8 \alpha_{s}^{2}} \beta\left(\alpha_{s}\right)\left\langle\frac{\alpha_{s}}{\pi} G_{\mu \nu} G^{\mu \nu}\right\rangle
$$




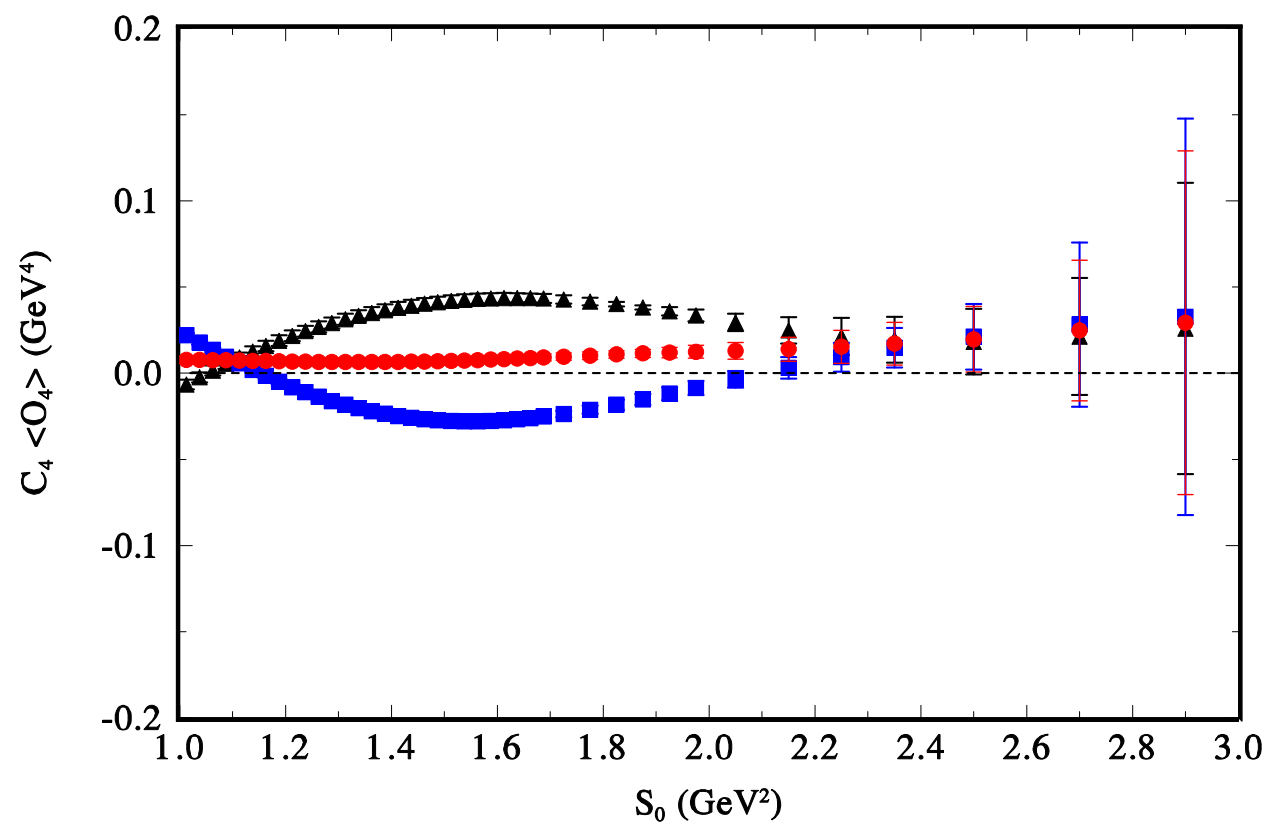

Figure 2. The dimension $d=4$ condensate in CIPT from the FESR, eq. (2.15), with $N=1$ and $\alpha_{s}\left(M_{\tau}^{2}\right)=0.341$. The ALEPH data for the $V$ (upper black dots), $A$ (lower blue dots) and $\frac{1}{2}(V+A)$ (middle red dots) spectral function were used.

Therefore, the sign and magnitude of the gluon condensate $\left\langle\frac{\alpha_{s}}{\pi} G_{\mu \nu} G^{\mu \nu}\right\rangle$ are of fundamental importance for the understanding of the strong interactions. A negative value of $\varepsilon$ is expected from models such as the bag model. In our analysis we obtain for $\alpha_{s}\left(M_{\tau}^{2}\right)=0.341$

$$
C_{4}\left\langle O_{4}\right\rangle=(0.017 \pm 0.012) \mathrm{GeV}^{4},
$$

where this value is obtained by reading results from the $V+A$ spectral function at $s_{0}=$ $2.35 \mathrm{GeV}^{2}$, i.e. at the point where $C_{4}\left\langle O_{4}\right\rangle$ from the $V$ and $A$ channels become equal. This value is consistent within errors with the points at higher values of $s_{0}$ and agrees with a previous determination [17] using the original ALEPH data base [2]. However, the uncertainty is now larger due to the new ALEPH error correlation matrix. We observe that the precise value for $\alpha_{s}$ chosen in the evaluation of the condensate has a relatively large impact on the result: the uncertainty of \pm 0.013 for $\alpha_{s}\left(M_{\tau}^{2}\right)$ given in [5] gives rise to an additional uncertainty of \pm 0.018 for $C_{4}\left\langle O_{4}\right\rangle$. We repeated the analysis using FOPT. The results are very similar, though. For example, for the central FOPT value $\alpha_{s}\left(M_{\tau}^{2}\right)=0.319$ we obtain $C_{4}\left\langle O_{4}\right\rangle=(0.022 \pm 0.006) \mathrm{GeV}^{4}$. Combining results, we can say that all evidence points to a positive value of $C_{4}\left\langle O_{4}\right\rangle \lesssim 0.035 \mathrm{GeV}^{4}$ which is equal for the vector and the axialvector correlators. In contrast, in the updated analysis of the ALEPH data [4] unequal and negative results for the $V$ and $A$ channels have been obtained.

The next condensates, i.e. with dimension $d=6$, in the vector and the axial-vector channels do not show a stability region. This type of FESR is not suited to extract higher dimensional condensates because the power weight in the FESR increasingly emphasizes the high energy region, where experimental errors are large and where the condensates are the result of a fine balanced cancellation between the hadronic integral and the PQCD 


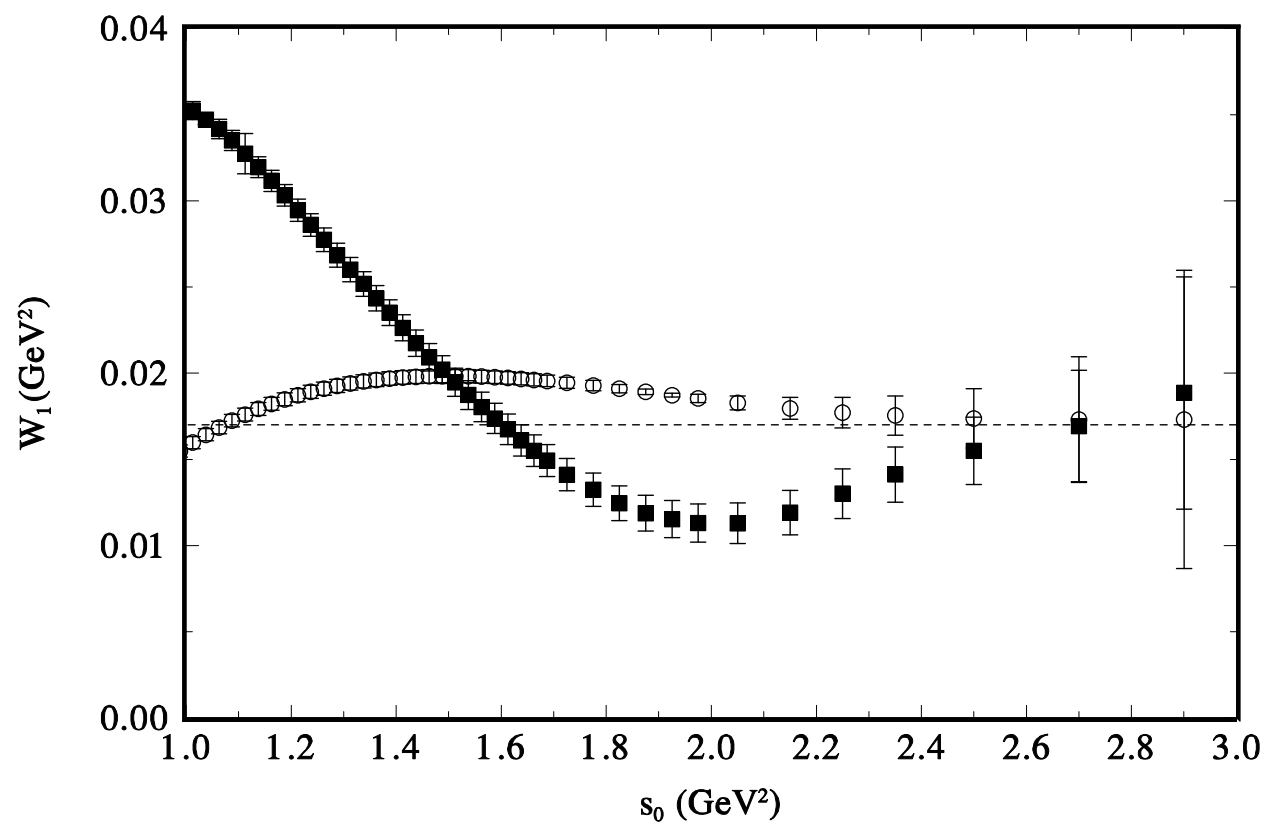

Figure 3. Solid squares are the left-hand-side of the standard Weinberg sum rule, eq. (3.1), and open circles the left hand side of the pinched sum rule, eq. (3.3). The dotted line is the right-handside, $2 f_{\pi}^{2}$.

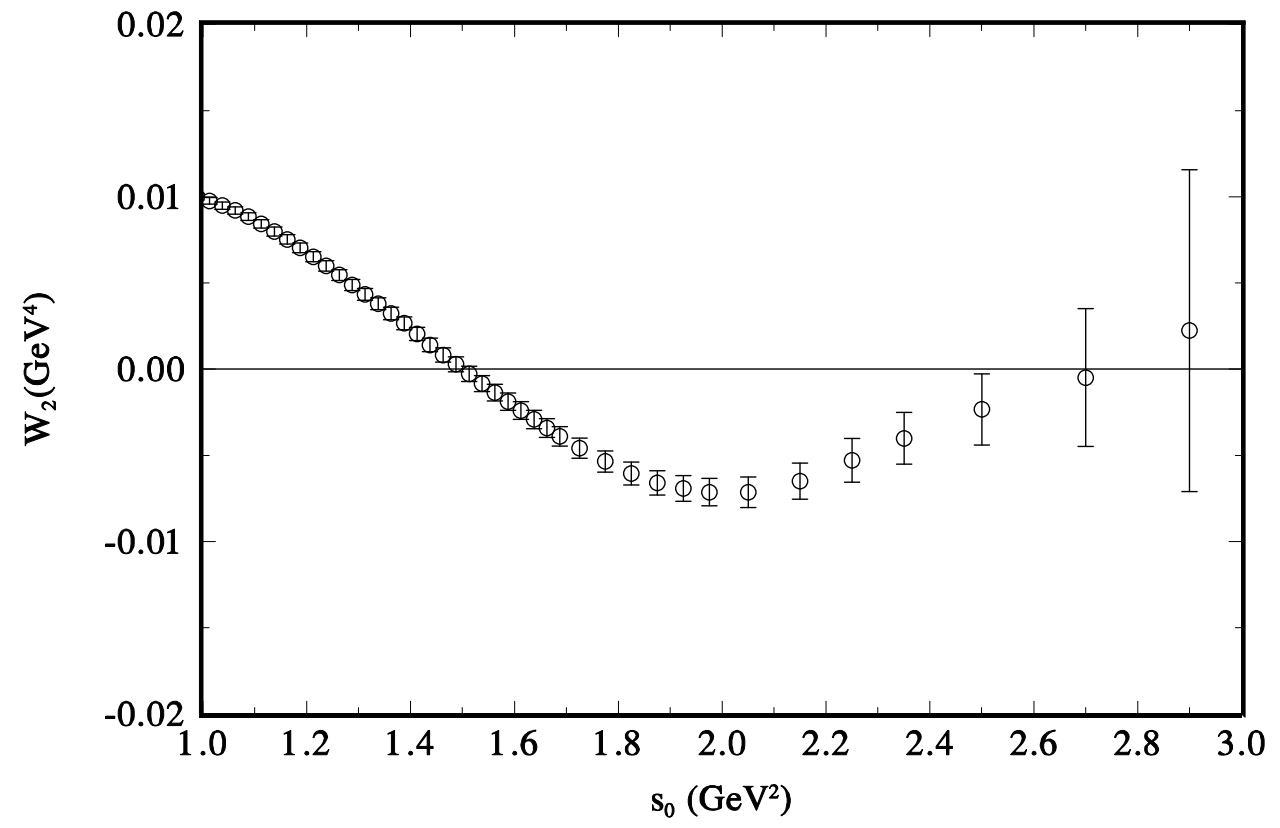

Figure 4. The second Weinberg sum rule $W_{2}$ as a function the upper limit of integration.

moments, with a marginally meaningful result at $d=4$, but not beyond. In the next section we shall determine the chiral condensates of dimension $d=6$ and $d=8$, which do not suffer from this handicap as the perturbative contribution cancels exactly (in the chiral limit). 


\section{Chiral sum rules and chiral vacuum condensates}

The two Weinberg sum rules (WSR) [41] were first derived in the framework of chiral $\mathrm{SU}(2) \times \mathrm{SU}(2)$ symmetry and current algebra, retaining their validity in QCD in the chiral limit, and read

$$
\begin{gathered}
W_{1} \equiv \int_{0}^{\infty} d s \frac{1}{\pi}\left[\operatorname{Im}_{V}(s)-\operatorname{Im}_{A}(s)\right]=2 f_{\pi}^{2}, \\
W_{2} \equiv \int_{0}^{\infty} d s s \frac{1}{\pi}\left[\operatorname{Im} \Pi_{V}(s)-\operatorname{Im}_{A}(s)\right]=0,
\end{gathered}
$$

where $f_{\pi}=92.21 \pm 0.14 \mathrm{MeV}$ [37]. The integration region can be split into two parts, one in the range $0-s_{0}$ and the other in $s_{0}-\infty$. Since the spectral function difference vanishes in PQCD for $s_{0}$ sufficiently large, these sum rules effectively become FESR. However, as pointed out long ago $[19,21]$, the original $\tau$-decay ALEPH data [2] did not saturate these integrals up to the kinematic end point $s_{0} \simeq M_{\tau}^{2}$. This could also be said for the updated ALEPH data [4] if the existence of a plateau of the central values is taken as a criterion for saturation (see solid squares in figure 3 for $W_{1}$ and open circles in figure 4 for $W_{2}$ ). The size of the experimental uncertainties, however, does not allow us to conclude that saturation has not been reached. A much better behaviour is achieved after introducing a simple pinched kernel and combining the two sum rules into one

$$
W_{1 P}\left(s_{0}\right) \equiv \int_{0}^{s_{0}} d s\left(1-\frac{s}{s_{0}}\right) \frac{1}{\pi}\left[\operatorname{Im} \Pi_{V}(s)-\operatorname{Im}_{A}(s)\right]=2 f_{\pi}^{2} .
$$

The result is shown in figure 3 (open circles), indicating a very good saturation of the pinched sum rule. This supports the use of simple integration kernels, although DV could be channel or application dependent.

The two Weinberg sum rules are particularly interesting since they would not be satisfied if there were substantial DV present, i.e. non-perturbative contributions beyond perturbative QCD and OPE contributions. The issue of DV is indeed most prominent in the context of the $V-A$ correlator, since the perturbative component cancels out leaving a purely non-perturbative result. The two simple, i.e. un-pinched Weinberg sum rules agree with the OPE expectations only near the end of the decay spectrum $s_{0} \simeq 2.7 \mathrm{GeV}^{2}$. Because of experimental limitations, the errors are relatively large and no definite conclusions on the relevance of duality violations can be drawn in this case. The last two experimental points should be ignored in the discussion because they cannot be accommodated either by PQCD and the OPE or in models for DV . The pinched sum rule, however, is saturated beginning at $s_{0} \geq 2.2 \mathrm{GeV}^{2}$ and shows remarkable agreement with the prediction of $2 f_{\pi}^{2}$. No compelling evidence is seen for the existence of DV in this kinematic domain. We assert that for simple un-pinched Weinberg sum rules, possible DV are not required for $s_{0} \gtrsim 2.7 \mathrm{GeV}^{2}$ while for the pinched sum rule possible DV can be ignored beginning at much lower momentum transfers, i.e. already for $s_{0} \gtrsim 2.2 \mathrm{GeV}^{2}$. In view of our result it seems very reasonable to take over this conclusion to the separate $V$ and $A$ sum rules. The lack of evidence for DV in the separate $V$ and $A$ correlators at large $s_{0}$ was also demonstrated in [47], albeit with the old ALEPH data. These conclusions are in contrast with those following from specific models of DV [22, 23, 25]. 


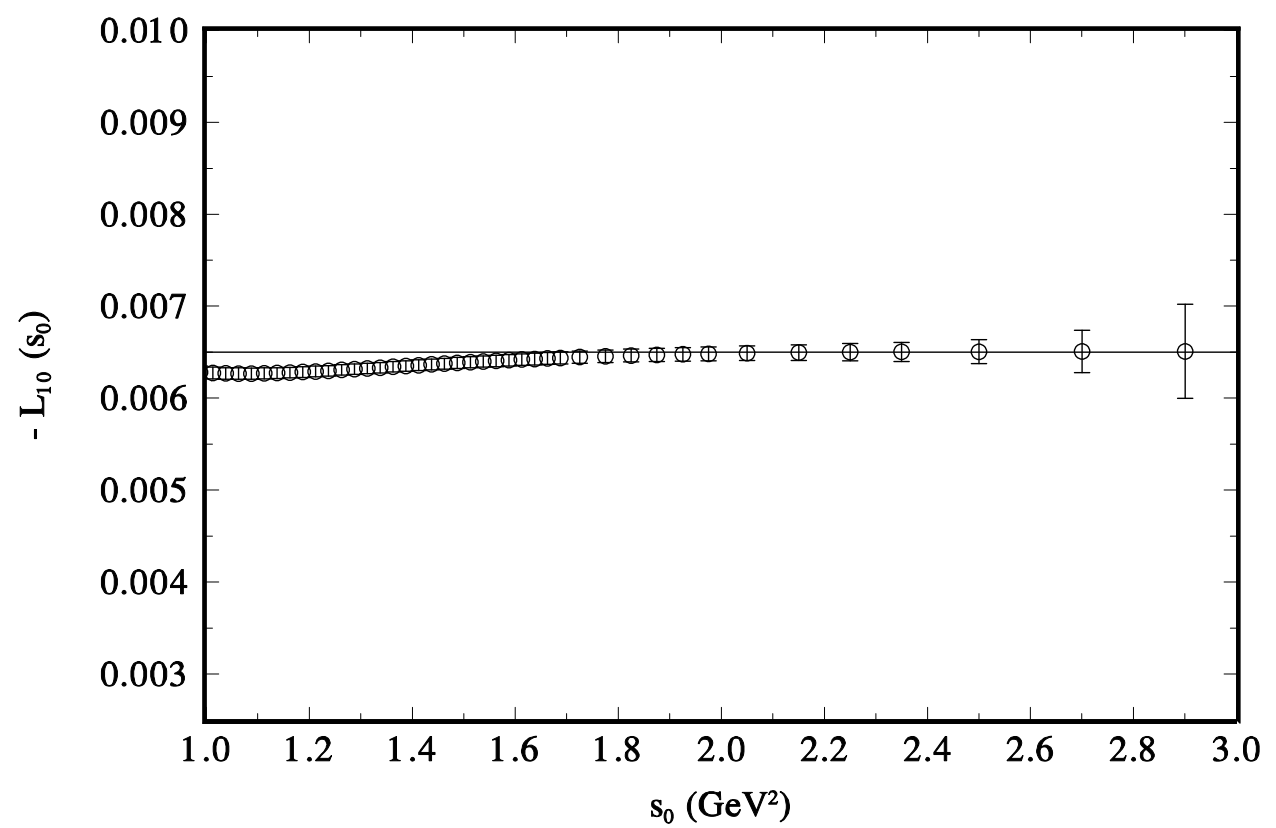

Figure 5. The CHPT constant $-\bar{L}_{10}$ obtained from the pinched chiral sum rule for $\bar{\Pi}(0)$ eq. (3.5).

Next, we consider the chiral correlator $\left.\Pi\left(Q^{2}\right)\right|_{V-A}$, and absorb the Wilson coefficients entering eq. (2.8) into the operators, renaming them $\mathcal{O}_{N}$ to conform with a usual convention in the literature,

$$
\left.\Pi\left(Q^{2}\right)\right|_{V-A}=\sum_{N=1}^{\infty} \frac{1}{Q^{2 N+4}}\left\langle\mathcal{O}_{2 N+4}\right\rangle,
$$

with the first two chiral condensates being $\left\langle\mathcal{O}_{6}\right\rangle$ and $\left\langle\mathcal{O}_{8}\right\rangle$. Dropping the label $V-A$, the finite remainder of this chiral correlator at zero momentum, $\bar{\Pi}(0)$, is given by

$$
\bar{\Pi}(0)=\int_{0}^{s_{0}} \frac{d s}{s} \frac{1}{\pi}\left[\operatorname{Im} \Pi_{V}(s)-\operatorname{Im}_{A}(s)\right],
$$

where $\operatorname{Im}_{A}(s)$ does not include the pion pole.

The chiral correlator at zero momentum, $\bar{\Pi}(0)$, is determined by the Das-MathurOkubo (DMO) sum rule [24],

$$
\bar{\Pi}(0)=2\left(\frac{1}{3} f_{\pi}^{2}\left\langle r_{\pi}^{2}\right\rangle-\frac{1}{2} F_{A}\right)=0.0520 \pm 0.0010,
$$

where $\left\langle r_{\pi}^{2}\right\rangle=0.439 \pm 0.008 \mathrm{fm}^{2}$ is the electromagnetic radius of the pion [42], and $F_{A}=$ $0.0119 \pm 0.0001$ is the radiative pion decay constant [37]. Since the numerical value on the right-hand side of eq. (3.6) is known with high precision, the DMO sum rule is another case where DV would easily become visible. Our results in figure 5 show a wide stability region starting already at $s_{0} \simeq 2 \mathrm{GeV}^{2}$ for the pinched DMO sum rule (using eqs. (3.1), (3.2))

$$
\bar{\Pi}(0)=4 \frac{f_{\pi}^{2}}{s_{0}}+\int_{0}^{s_{0}} \frac{d s}{s}\left(1-\frac{s}{s_{0}}\right)^{2} \frac{1}{\pi}\left[\operatorname{Im} \Pi_{V}(s)-\operatorname{Im}_{A}(s)\right] .
$$




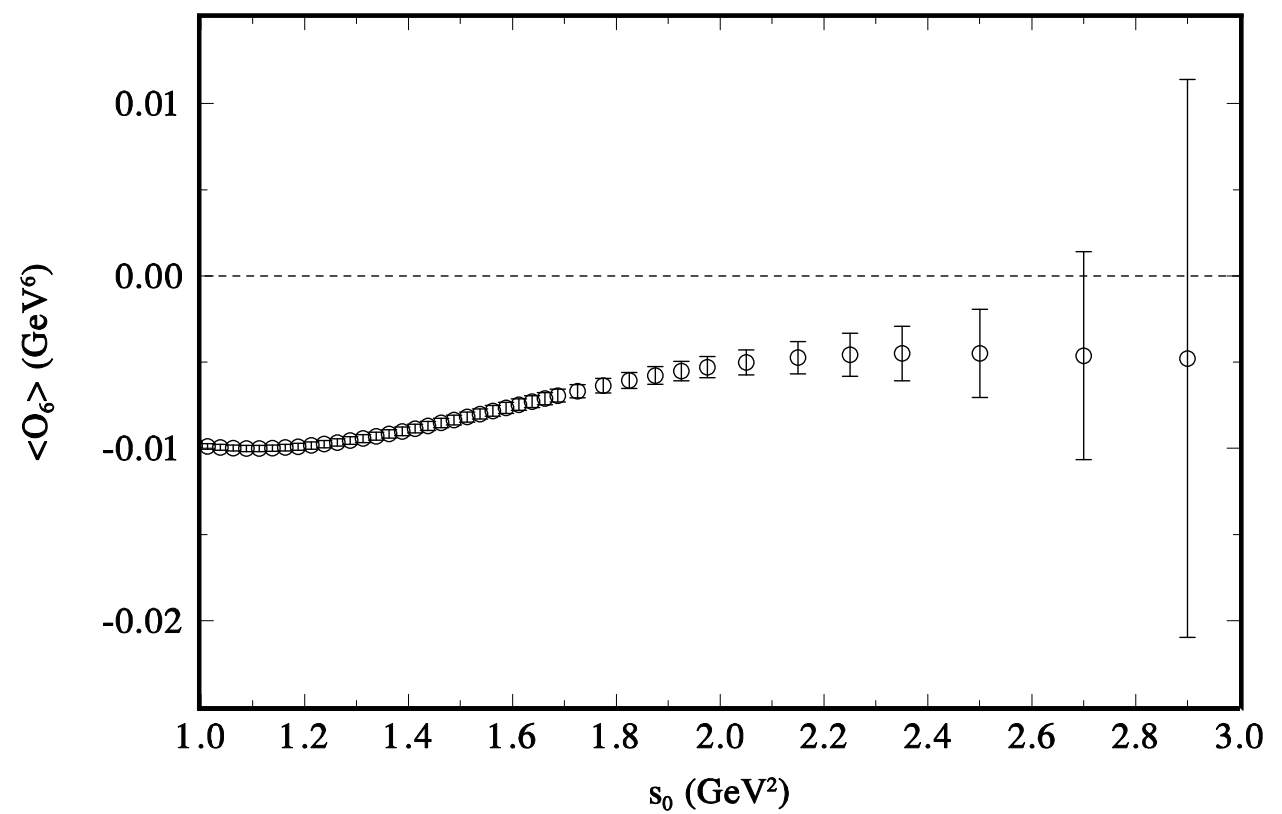

Figure 6. The chiral condensate of dimension $d=6$ from the pinched chiral sum rule eq. (3.10).

$\bar{\Pi}(0)$ is proportional to the counter term of the order $\mathcal{O}\left(p^{4}\right)$ Lagrangian of chiral perturbation theory, $\bar{L}_{10}[43,44]$,

$$
\bar{\Pi}(0)=-8 \bar{L}_{10}
$$

We find

$$
\bar{L}_{10}=-(6.5 \pm 0.1) \times 10^{-3} .
$$

This result is in very good agreement with an early determination based on the original ALEPH data base [21], $\bar{L}_{10}=-(6.43 \pm 0.08) \times 10^{-3}$, as well as with more recent results using more involved methods to deal with DV , e.g. $\bar{L}_{10}=-(6.46 \pm 0.15) \times 10^{-3}$ from $[22,23]$, and $\bar{L}_{10}=-(6.52 \pm 0.14) \times 10^{-3}$ from [25]. It also agrees with lattice QCD determinations within their larger uncertainties $[45,46]$.

The relation between $\bar{\Pi}(0)$ and the precisely known quantities $f_{\pi}^{2},\left\langle r_{\pi}^{2}\right\rangle$ and $F_{A}$, is another case where the presence of DV can be tested. We observe that our result shown in figure 5 is very stable with respect to variations of $s_{0}$ in the range above $2 \mathrm{GeV}^{2}$ and the result from CHPT is reproduced with amazingly good accuracy.

Turning to the chiral condensates, for dimension $d=6$ we use the following pinched FESR [21]

$$
\left\langle\mathcal{O}_{6}\right\rangle=-2 f_{\pi}^{2} s_{0}^{2}+s_{0}^{2} \int_{0}^{s_{0}} d s\left(1-\frac{s}{s_{0}}\right)^{2} \frac{1}{\pi}\left[\operatorname{Im} \Pi_{V}(s)-\operatorname{Im}_{A}(s)\right] .
$$

The result is shown in figure 6. Stability is observed for $s_{0} \gtrsim 2 \mathrm{GeV}^{2}$. Assuming that DV are not relevant in this kinematic range, we read off the value

$$
\left\langle\mathcal{O}_{6}\right\rangle=-(5.0 \pm 0.7) \times 10^{-3} \mathrm{GeV}^{6}
$$




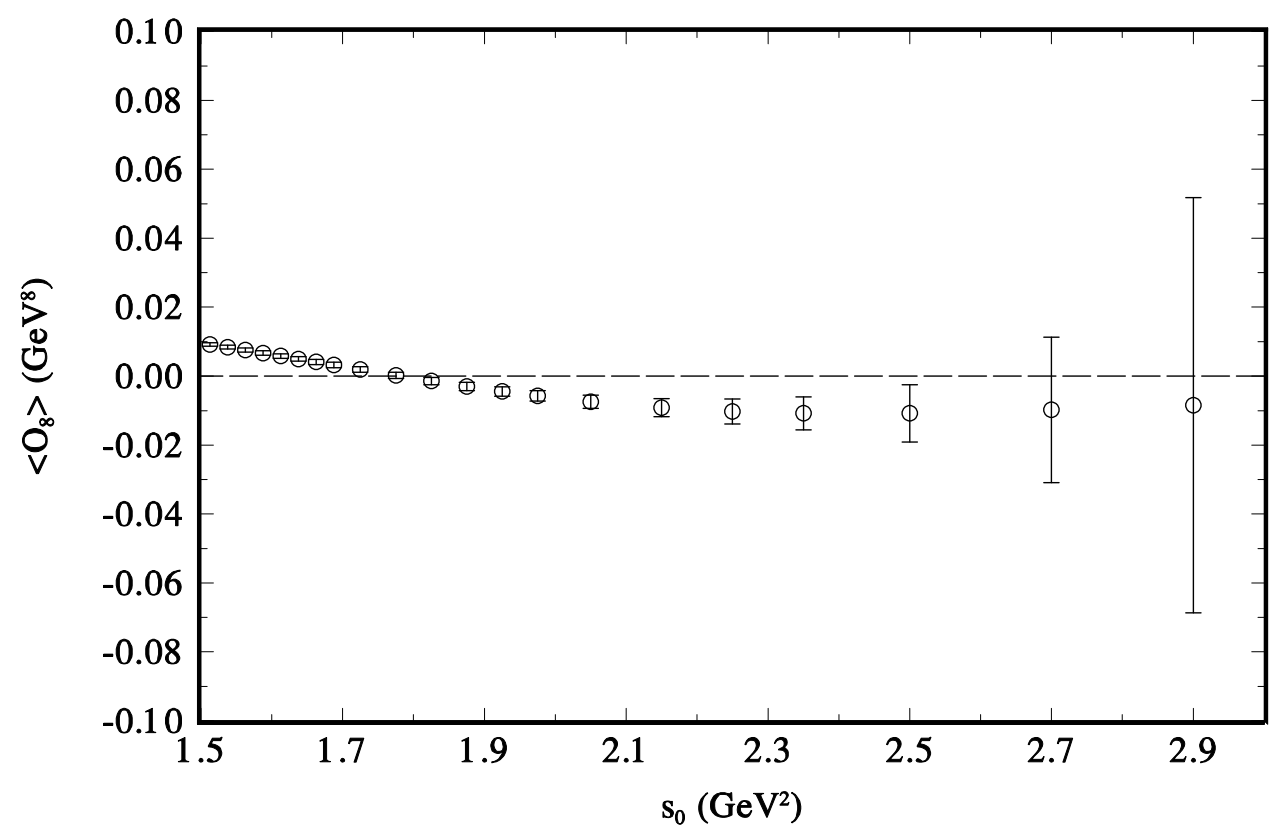

Figure 7. The chiral condensate of dimension $d=8$ from the pinched chiral sum rule eq. (3.13).

This value agrees with [21] obtained from the same sum rule, eq. (3.10), but using the original ALEPH data, i.e. $\left\langle\mathcal{O}_{6}\right\rangle=-(4.0 \pm 1.0) \times 10^{-3} \mathrm{GeV}^{6}$. It also agrees with [22, 23], i.e. $\left\langle\mathcal{O}_{6}\right\rangle=-(4.3 \pm 0.9) \times 10^{-3} \mathrm{GeV}^{6}$, as well as with [25] $\left\langle\mathcal{O}_{6}\right\rangle=-(6.6 \pm 1.1) \times 10^{-3} \mathrm{GeV}^{6}$. In addition, this value agrees within errors with the four-quark condensate in the vacuumsaturation approximation [48]

$$
\left.\left\langle\mathcal{O}_{6}\right\rangle\right|_{V S}=-\frac{64 \pi}{9} \alpha_{s}\langle\bar{q} q\rangle^{2}\left[1+\frac{247}{48 \pi} \alpha_{s}\left(s_{0}\right)\right] \simeq-4.6 \times 10^{-3} \mathrm{GeV}^{6} .
$$

Finally, we determine the $d=8$ chiral condensate from the pinched sum rule [21]

$$
\left\langle\mathcal{O}_{8}\right\rangle=16 f_{\pi}^{2} s_{0}^{3}-3 s_{0}^{4} \bar{\Pi}(0)+s_{0}^{3} \int_{0}^{s_{0}} \frac{d s}{s}\left(1-\frac{s}{s_{0}}\right)^{3}\left(s+3 s_{0}\right) \frac{1}{\pi}\left[\operatorname{Im} \Pi_{V}(s)-\operatorname{Im}_{A}(s)\right] .
$$

The result is shown in figure 7 , which leads to

$$
\left\langle\mathcal{O}_{8}\right\rangle=-(9.0 \pm 5.0) \times 10^{-3} \mathrm{GeV}^{8},
$$

a considerably more accurate value than that of $[21],\left\langle\mathcal{O}_{8}\right\rangle=-(1.0 \pm 6.0) \times 10^{-3} \mathrm{GeV}^{8}$, as well as that of $[25]\left\langle\mathcal{O}_{8}\right\rangle=(5.0 \pm 5.0) \times 10^{-3} \mathrm{GeV}^{8}$. The present result does agree within errors with that of $[22,23]\left\langle\mathcal{O}_{8}\right\rangle=-(7.2 \pm 4.8) \times 10^{-3} \mathrm{GeV}^{8}$.

\section{Conclusion}

The new ALEPH data base [4] has been used together with QCD FESR to redetermine a potential dimension $d=2$ term in the OPE, as well as the dimension $d=4$ vacuum condensate, i.e. the gluon condensate in the chiral limit. The former term is consistent 
with zero, thus confirming expectations [16], as well as previous results [17], while the latter is affected by a larger uncertainty than the result from the original ALEPH data base [17]. It is important to notice that the current uncertainty in the strong coupling (at the scale of the $\tau$-lepton mass) dominates over the data errors in the final uncertainty in the condensates as obtained from FESR.

The two Weinberg sum rules are saturated by the data at the end of the $\tau$-decay spectrum. A simple pinched combination of the Weinberg sum rules as well as the DasMathur-Okubo sum rule have turned out to be amazingly well saturated at much lower center-of-mass energies. We consider this as an indication that DV are not needed. We are not asserting, though, that DV do not exist. Instead, we interpret this good saturation as suggesting that our pinched kernels might have quenched any potential DV. However, this conclusion is not universally accepted $[22,23,25]$. Given the unavoidable need of specific models to account for the postulated DV, this issue remains currently an open problem. Similar pinched integration kernels were then used here to determine the chiral correlator at zero momentum, as well as the chiral condensates of dimension $d=6$ and $d=8$. In comparison with results using the original ALEPH data base, the major changes are in the values of the gluon condensate and of the chiral condensates.

\section{Acknowledgments}

This work was supported in part by DFG (Germany), and by NRF and NITheP (South Africa). One of us (CAD) wishes to thank Andrew Hamilton for a discussion on the error analysis.

Open Access. This article is distributed under the terms of the Creative Commons Attribution License (CC-BY 4.0), which permits any use, distribution and reproduction in any medium, provided the original author(s) and source are credited.

\section{References}

[1] ARGUS collaboration, H. Albrecht et al., Measurement of $\tau$ decays into three charged pions, Z. Phys. C 33 (1986) 7 [InSPIRE].

[2] ALEPH collaboration, R. Barate et al., Measurement of the spectral functions of axial-vector hadronic tau decays and determination of $\alpha_{s}\left(M_{\tau}^{2}\right)$, Eur. Phys. J. C 4 (1998) 409 [INSPIRE].

[3] ALEPH collaboration, S. Schael et al., Branching ratios and spectral functions of $\tau$ decays: final ALEPH measurements and physics implications, Phys. Rept. 421 (2005) 191 [hep-ex/0506072] [INSPIRE].

[4] M. Davier, A. Höcker, B. Malaescu, C.-Z. Yuan and Z. Zhang, Update of the ALEPH non-strange spectral functions from hadronic $\tau$ decays, Eur. Phys. J. C 74 (2014) 2803 [arXiv: 1312.1501] [INSPIRE].

[5] A. Pich, Precision tau physics, Progr. Part. Nucl. Phys. 75 (2014) 41 [arXiv:1310.7922] [INSPIRE]. 
[6] R.A. Bertlmann, C.A. Dominguez, M. Loewe, M. Perrottet and E. de Rafael, Determination of the gluon condensate and the four quark condensate via FESR, Z. Phys. C 39 (1988) 231 [INSPIRE].

[7] C.A. Dominguez and J. Solà, Determination of quark and gluon vacuum condensates from $\tau$ lepton decay data, Z. Phys. C 40 (1988) 63 [inSPIRE].

[8] V. Giménez, J.A. Penarrocha and J. Bordes, QCD condensates from $\tau$ decay experimental data in the axial channel, Phys. Lett. B 223 (1989) 245 [INSPIRE].

[9] J. Rojo and J.I. Latorre, Neural network parametrization of spectral functions from hadronic tau decays and determination of QCD vacuum condensates, JHEP 01 (2004) 055 [hep-ph/0401047] [INSPIRE].

[10] V. Cirigliano, E. Golowich and K. Maltman, $Q C D$ condensates for the light quark $V$-A correlator, Phys. Rev. D 68 (2003) 054013 [hep-ph/0305118] [INSPIRE].

[11] B.V. Geshkenbein, Hadronic tau decay, the renormalization group, analyticity of the polarization operators and QCD parameters, Phys. Rev. D 67 (2003) 074006 [hep-ph/0206094] [INSPIRE].

[12] M. Davier, A. Höcker and Z. Zhang, The physics of hadronic $\tau$ decays, Rev. Mod. Phys. 78 (2006) 1043 [hep-ph/0507078] [INSPIRE].

[13] J. Bordes, C.A. Dominguez, J. Penarrocha and K. Schilcher, Chiral condensates from tau decay: a critical reappraisal, JHEP 02 (2006) 037 [hep-ph/0511293] [INSPIRE].

[14] A.A. Almasy, K. Schilcher and H. Spiesberger, $Q C D$ condensates of dimension $D=6$ and $D=8$ from hadronic $\tau$-decays, Phys. Lett. B 650 (2007) 179 [hep-ph/0612304] [INSPIRE].

[15] A.A. Almasy, K. Schilcher and H. Spiesberger, Determination of QCD condensates from $\tau$-decay data, Eur. Phys. J. C 55 (2008) 237 [arXiv:0802.0980] [INSPIRE].

[16] C.A. Dominguez and K. Schilcher, Is there evidence for dimension two corrections in QCD two point functions?, Phys. Rev. D 61 (2000) 114020 [hep-ph/9903483] [InSPIRE].

[17] C.A. Dominguez and K. Schilcher, QCD vacuum condensates from tau-lepton decay data, JHEP 01 (2007) 093 [hep-ph/0611347] [INSPIRE].

[18] P. Colangelo and A. Khodjamirian, QCD sum rules, a modern approach, in At the frontier of particle physics/Handbook of QCD, volume 3, M. Shifman ed., World Scientific, Singapore (2001).

[19] C.A. Dominguez and K. Schilcher, Chiral sum rules and duality in QCD, Phys. Lett. B 448 (1999) 93 [hep-ph/9811261] [INSPIRE].

[20] M. Gell-Mann, R.J. Oakes and B. Renner, Behavior of current divergences under $\mathrm{SU}(3) \times \mathrm{SU}(3)$, Phys. Rev. 175 (1968) 2195 [inSPIRE].

[21] C.A. Dominguez and K. Schilcher, Finite energy chiral sum rules in $Q C D$, Phys. Lett. B 581 (2004) 193 [hep-ph/0309285] [INSPIRE].

[22] M. Gonzalez-Alonso, A. Pich and J. Prades, Violation of quark-hadron duality and spectral chiral moments in QCD, Phys. Rev. D 81 (2010) 074007 [arXiv:1001.2269] [INSPIRE].

[23] M. Gonzalez-Alonso, A. Pich and J. Prades, Pinched weights and duality violations in QCD sum rules: a critical analysis, Phys. Rev. D 82 (2010) 014019 [arXiv: 1004.4987] [INSPIRE].

[24] T. Das, V.S. Mathur and S. Okubo, Low-energy theorem in the radiative decays of charged pions, Phys. Rev. Lett. 19 (1967) 859 [InSPIRE]. 
[25] D. Boito, M. Golterman, M. Jamin, K. Maltman and S. Peris, Low-energy constants and condensates from the $\tau$ hadronic spectral functions, Phys. Rev. D 87 (2013) 094008 [arXiv: 1212.4471] [INSPIRE].

[26] K. Schilcher and M.D. Tran, Duality in semileptonic $\tau$ decay, Phys. Rev. D 29 (1984) 570 [INSPIRE].

[27] M. Golterman, K. Maltman and S. Peris, NNLO low-energy constants from flavor-breaking chiral sum rules based on hadronic $\tau$-decay data, Phys. Rev. D 89 (2014) 054036 [arXiv: 1402.1043] [INSPIRE].

[28] D. R. Boito et al., Duality violations in tau hadronic spectral moments, Nucl. Phys. Proc. Suppl. 218 (2011) 104 [arXiv:1011.4426] [InSPIRE].

[29] K.G. Chetyrkin, A.L. Kataev and F.V. Tkachov, Higher order corrections to $\Sigma_{t}\left(e^{+} e^{-} \rightarrow\right.$ hadrons) in quantum chromodynamics, Phys. Lett. B 85 (1979) 277 [INSPIRE].

[30] M. Dine and J.R. Sapirstein, Higher order QCD corrections in $e^{+} e^{-}$annihilation, Phys. Rev. Lett. 43 (1979) 668 [INSPIRE].

[31] W. Celmaster and R.J. Gonsalves, An analytic calculation of higher order quantum chromodynamic corrections in $e^{+} e^{-}$annihilation, Phys. Rev. Lett. 44 (1980) 560 [INSPIRE].

[32] S.G. Gorishnii, A.L. Kataev and S.A. Larin, The $O\left(\alpha_{S}^{3}\right)$ corrections to $\Sigma_{t}\left(e^{+} e^{-} \rightarrow\right.$ hadrons $)$ and $\Gamma\left(\tau^{-} \rightarrow \tau\right.$-neutrino + hadrons) in QCD, Phys. Lett. B 259 (1991) 144 [inSPIRE].

[33] L.R. Surguladze and M.A. Samuel, Total hadronic cross-section in $e^{+} e^{-}$annihilation at the four loop level of perturbative QCD, Phys. Rev. Lett. 66 (1991) 560 [Erratum ibid. 66 (1991) 2416] [INSPIRE].

[34] R. Shankar, Determination of the quark-gluon Coupling Constant, Phys. Rev. D 15 (1977) 755 [INSPIRE].

[35] K. Maltman, Constraints on hadronic spectral functions from continuous families of finite energy sum rules, Phys. Lett. B 440 (1998) 367 [hep-ph/9901239] [INSPIRE].

[36] P.A. Baikov, K.G. Chetyrkin and J.H. Kuhn, Order $\alpha_{s}^{4} Q C D$ corrections to $Z$ and $\tau$ decays, Phys. Rev. Lett. 101 (2008) 012002 [arXiv:0801.1821] [INSPIRE].

[37] Particle Data Group collaboration, J. Beringer et al., Review of particle physics, Phys. Rev. D 86 (2012) 010001 [inSPIRE].

[38] W.J. Marciano and A. Sirlin, Electroweak radiative corrections to $\tau$ decay, Phys. Rev. Lett. 61 (1988) 1815 [inSPIRE].

[39] M.A. Shifman, A.I. Vainshtein and V.I. Zakharov, QCD and resonance physics. Sum rules, Nucl. Phys. B 147 (1979) 385 [inSPIRE].

[40] M.A. Shifman, A.I. Vainshtein and V.I. Zakharov, QCD and resonance physics: applications, Nucl. Phys. B 147 (1979) 448 [inSPIRE].

[41] S. Weinberg, Precise relations between the spectra of vector and axial vector mesons, Phys. Rev. Lett. 18 (1967) 507 [INSPIRE].

[42] NA7 collaboration, S.R. Amendolia et al., A measurement of the space-like pion electromagnetic form-factor, Nucl. Phys. B 277 (1986) 168 [INSPIRE].

[43] J. Gasser and H. Leutwyler, Chiral perturbation theory: expansions in the mass of the strange quark, Nucl. Phys. B 250 (1985) 465 [INSPIRE]. 
[44] G. Ecker, J. Gasser, A. Pich and E. de Rafael, The role of resonances in chiral perturbation theory, Nucl. Phys. B 321 (1989) 311 [InSPIRE].

[45] JLQCD collaboration, E. Shintani et al., S-parameter and pseudo-Nambu-Goldstone boson mass from lattice QCD, Phys. Rev. Lett. 101 (2008) 242001 [arXiv:0806.4222] [INSPIRE].

[46] RBC, UKQCD collaboration, P.A. Boyle, L. Del Debbio, J. Wennekers and J.M. Zanotti, The S parameter in QCD from domain wall fermions, Phys. Rev. D 81 (2010) 014504 [arXiv:0909.4931] [INSPIRE].

[47] C.A. Dominguez, N.F. Nasrallah and K. Schilcher, Confronting QCD with the experimental hadronic spectral functions from $\tau$-decay, Phys. Rev. D 80 (2009) 054014 [arXiv:0903.3463] [INSPIRE].

[48] L.V. Lanin, V.P. Spidorov and K.G. Chetyrkin, On the contribution of the four-quark condensate to the sum rules for the rho and $A_{1}$ mesons, Sov. J. Nucl. Phys. 44 (1986) 892. 